\title{
Force measurements with optical tweezers inside living cells
}

\author{
Josep Mas ${ }^{\mathrm{a}, \mathrm{b}}$, Arnau Farré, ${ }^{\mathrm{a}, \mathrm{c}}$, Jordi Sancho-Parramon ${ }^{\mathrm{a}, \mathrm{d}}$, Estela Martín-Badosa ${ }^{\mathrm{a}}$, Mario Montes- \\ Usategui*a,c \\ ${ }^{a}$ Departament de Física Aplicada i Òptica, Universitat de Barcelona, Spain \\ ${ }^{b}$ Department of Physics and Astronomy, University of Saint Andrews, United Kingdom \\ 'Impetux Optics SL, Barcelona, Spain \\ ${ }^{\mathrm{d}}$ Ruđer Bošković Institute, Zagreb, Croatia.
}

\begin{abstract}
The force exerted by optical tweezers can be measured by tracking the momentum changes of the trapping beam, a method which is more general and powerful than traditional calibration techniques as it is based on first principles, but which has not been brought to its full potential yet, probably due to practical difficulties when combined with high-NA optical traps, such as the necessity to capture a large fraction of the scattered light. We show that it is possible to measure forces on arbitrary biological objects inside cells without an in situ calibration, using this approach. The instrument can be calibrated by measuring three scaling parameters that are exclusively determined by the design of the system, thus obtaining a conversion factor from volts to piconewtons that is theoretically independent of the physical properties of the sample and its environment. We prove that this factor keeps valid inside cells as it shows good agreement with other calibration methods developed in recent years for viscoelastic media. Finally, we apply the method to measuring the stall forces of kinesin and dynein in living A549 cells.
\end{abstract}

Keywords: optical tweezers, force measurements, molecular motors, cell biology

\section{INTRODUCTION}

Cells are the basic unit of organization of all living matter. Since their discovery in the $17^{\text {th }}$ century their study and understanding has been linked to advances in microscopy techniques. Cells, although enormously varied, share a common chemical composition and similar organization principles, which are becoming to be accessible to individual molecular scrutiny only recently through modern single-molecule biophysics techniques. Among these, optical tweezers stand out for their capacity to exert forces in a key range of $0.1-100 \mathrm{pN}$, which are typical of many cellular phenomena. For example, optical trapping assays have determined the stall force and the discrete stepping behavior of the kinesin molecular motors ${ }^{1}$, have unraveled the dynamics and force generation capabilities of microtubule bundles ${ }^{2}$, or have provided invaluable information on the mechanical properties of biological membranes ${ }^{3}$.

Most optical trapping experiments consist of assays in vitro with reconstituted elements previously isolated and purified. This allowed to set bounds to the complexity of cell's biochemical mechanisms and thus permitted to dissect in an effective way these processes. However, this advantage is also a curse and the information gained in these experiments is widely acknowledged as partial. The motor protein dynein is a prominent example of the incompleteness of our current information in vitro. Recent experiments in vivo have shown that dynein is capable of sharing loads much more efficiently than kinesin by quickly reducing its velocity under load, and contrary to kinesin, dynein can bind tenaciously to the microtubule when close to its stall force, by means of a "catch-bond" mechanism ${ }^{4}$. This contextualizes the small forces displayed by dynein in several motility assays in vitro $(\sim 1.1 \mathrm{pN})$. Furthermore, the value of the stall force in vitro is itself controversial ${ }^{5,6}$ (either $\sim 1.1 \mathrm{pN}$ or $\sim 7 \mathrm{pN}$ ) and there are recent reports, themselves contradictory, of its value in vivo, which differ from the two values above (3-4 pN) (see section 4).

A combination of bottom-up (building more sophisticated in vitro assays) and top-down approaches (further studying these mechanisms in the living cell) is necessary to advance our understanding of these processes ${ }^{7}$. 
Unfortunately, the calibration of optical traps, which is reasonably straightforward in the viscous buffers employed in single-molecule assays, presents difficulties in vivo or in complex media that may recreate the cytoplasm in vitro. Some attempts to measure forces in cells have relied on extrapolations of a prior calibration done on purified vesicles, in a buffer that reproduced the index of refraction of the cytoplasm ${ }^{4}$. Methods better grounded in theory, specifically targeted to calibrate optical traps in a viscoelastic medium such as the cytoplasm are few ${ }^{8,9}$ and only recently have started to be implemented and validated.

Here we discuss an alternative route to perform these measurements based on the determination of the momentum changes of the trapping beam ${ }^{10-13}$. Our approach is based on very general physical principles, does not rely on the description of the Langevin dynamics of the trapped particle and therefore does not necessitate data of the sample or its environment. We show that the force scale is totally linked to a set of three parameters related to physical, optical or electrical characteristics of the measuring instrument, which, as a consequence, has to be determined only once, being this calibration valid for measuring on any sample. The extreme simplicity of operation, once the system has been built and calibrated, allowed us to measure a large number of stall forces of dynein and kinesin in living A549 cells. Our results are consistent with previous works that reported in vivo stall forces that differ from in vitro values (both kinesin and dynein stalling at 3-4 pN).

\section{THE MOMENTUM METHOD: A PERMANENT CALIBRATION}

The momentum method was devised by S. Smith and C. Bustamante ${ }^{10}$ at around the same time as back-focal plane interferometry (1996), but has been employed much less frequently despite its several advantages, due to difficulties in its practical implementation for high-NA traps. The method monitors the momentum changes of photons in the trapping beam as these go through the sample. The momentum information is contained in the angular intensity, $I(\theta, \phi)$, distribution according to ${ }^{11}$ :

$$
\vec{F}=\frac{n}{c} \lim _{r \rightarrow \infty} \int_{A}\left(\vec{S}_{T}-\vec{S}_{i}\right) d A=\frac{n}{4 \pi c} \int_{A} I(\theta, \phi) \hat{r} d \gamma
$$

where $\mathrm{n}$ is the index of refraction of the suspension medium, c the speed of light, $\mathrm{S}_{\mathrm{T}}$ and $\mathrm{S}_{\mathrm{i}}$ represent the Poynting vectors entering and leaving the surface of integration $\mathrm{A}, \mathrm{r}$ is unit vector from the focus and $\gamma$ represents a solid angle.

The angular light distribution $I(\theta, \phi)$ can be experimentally captured with enough accuracy by means of an appropriately high-NA lens ${ }^{12,13}$, properly projected at its back-focal plane if the lens fulfills the Abbe sine condition and can be there integrated by a position sensitive detector (PSD), which will produce signals proportional to the force. Despite the large variability of $I(\theta, \phi)$ (e. g. with sample size, Figure 1) the calibration factor $\alpha$ in this method is independent of the interaction between light and sample, as all light is captured (in contrast to back-focal plane interferometry). The value of the calibration constant $\alpha$ is determined by the following expression ${ }^{11}$ :

$$
F_{i}=\alpha V_{i}=\frac{R_{d}}{c f^{\prime} \Psi} V_{i}
$$

where $\mathrm{F}$ represents force (i.e. in $\mathrm{pN}$ ) and $\mathrm{V}$ the signal from the PSD (in volts). $\mathrm{R}_{\mathrm{d}}$ is the detector half-width, $\mathrm{c}$ the speed of light, f' the equivalent focal length of the capturing optics and $\Psi$ a responsivity parameter (in volts/watt) that relates the output of the PSD with the power at the sample.

We have previously shown that the momentum method can be used together with single beam optical traps despite the trapping light being scattered at large angles. Since a system based on momentum changes will also respond linearly to sample displacements, a link can be established between the calibration constant $\alpha$ and the product between the stiffness $\kappa$ and the position sensitivity $\beta$, according to: 

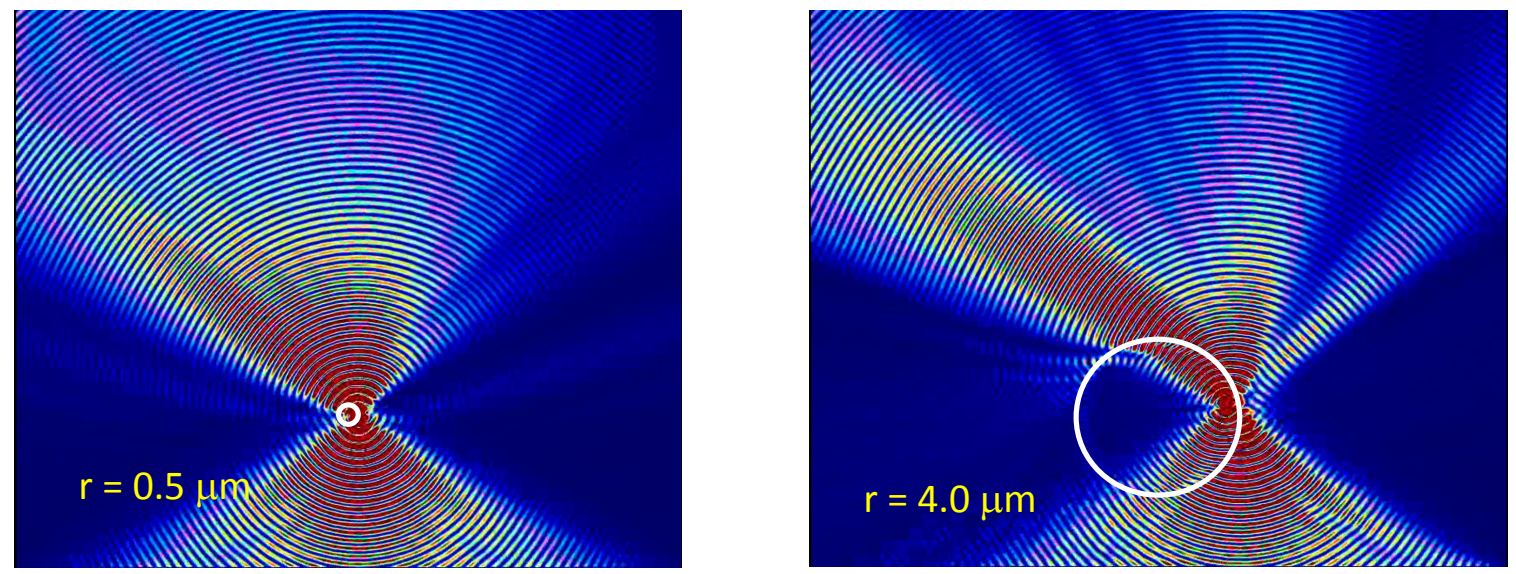

Figure 1. FDTD simulation of the angular intensity distribution of scattered light $I(\theta, \phi)$ for polystyrene beads of a) $\mathrm{r}=0.5$ $\mu \mathrm{m}$ and b) $\mathrm{r}=4.0 \mu \mathrm{m}$ (beam NA=1.2).

$$
F=-\kappa x=-\kappa \beta V=\alpha V,
$$

which can be verified through the power spectrum method.

Our prior results clearly proved that the calibration of the system does not depend on any characteristic of the sample or the trapping beam, being determined solely by the three parameters above ${ }^{13}\left(\mathrm{R}_{\mathrm{d}}, \mathrm{f}^{\prime}\right.$ and $\left.\Psi\right)$ (Fig. 2).

The complex microstructure of the cytoplasm and the a priori unknown properties of the cellular organelles (size and refractive index) make the calibration of optical traps in cells particularly challenging. A permanent calibration, on the contrary, would greatly simplify the measurements and make them more reliable. However, whether this method could still be accurately used inside a cell is unclear as the trapping beam will have go through a packed cytoplasm, which contains plenty of additional scattering structures, and cross the lipid membrane before it can be captured and analyzed.

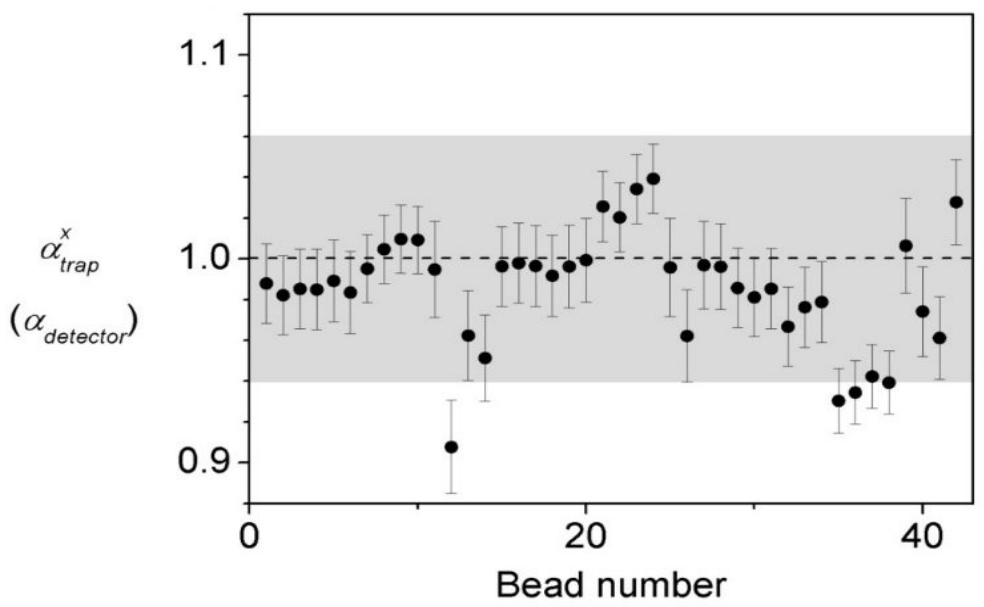

Figure 2. Comparison of calibration constant $\alpha$ measured according to equation $2\left(\mathrm{R}_{\mathrm{d}}=4.5 \mathrm{~mm}, \mathrm{f}^{\prime}=2.62 \mathrm{~mm}\right.$ and $\Psi=58$ $\mathrm{V} / \mathrm{W}$, normalized to 1 in the graph) with the product $\kappa \beta$ measured through the power spectrum method for a set of experimental conditions that involve variations in the size of the samples $(\mathrm{d}=0.61,1.16,2.19,3.06$ and $8.06 \mu \mathrm{m})$ and their refractive index $(\mathrm{n}=1.48,1.59,1.68)$, different trapping objectives $(\mathrm{NA}=1.2$, water immersion and $\mathrm{NA}=1.3$, oil immersion) as well as different trapping powers. The product keeps constant $(99 \mathrm{pN} / \mathrm{V})$ within a $6 \%$ error (adapted from reference 13). 
Following this rationale, we validated the light momentum method by comparing the value of the constant $\alpha$ determined from first principles (equation 2) with calibration experiments carried out in the cytoplasm by the active-passive method $^{8,9}$, in two different cell types: plant and mammalian cells.

\section{THE CALIBRATION CONSTANT KEEPS VALID INSIDE CELLS}

The active-passive calibration method (also known as the fluctuation-dissipation theorem method) was specifically developed in order to measure the stiffness of an optical trap $\kappa$, the positional calibration factor $\beta$, and additional information about the rheological properties of a viscoelastic medium ${ }^{8,9}$. The method considers a generalized Langevin equation that incorporates viscoelastic friction $\gamma_{1}$ and hydrodynamic memory $\gamma_{2}$ :

$$
m \ddot{x}_{u}(t)=-\int_{0}^{\infty} \gamma_{1, u}(\tau) \dot{x}_{u}(t-\tau) d \tau-\int_{0}^{\infty} \gamma_{2, u}(\tau) \ddot{x}_{u}(t-\tau) d \tau-\kappa x_{u}(t)+F_{\text {random }}(t)
$$

where $\mathrm{m}$ represents the mass of the trapped particle and $\mathrm{x}(\mathrm{t})$ position in time, and $\mathrm{F}_{\text {random }}$ stands for the stochastic thermal force.

Passive recordings of the power spectrum are now not enough for solving for the 4 unknowns: $\kappa, \gamma_{1}, \gamma_{2}$, and $\mathrm{m}$, so they have to be complemented with active perturbations of the system, for example by moving the trapped sample in a sinusoidal pattern of varying frequency, via a motorized stage. All these observations are then combined through Onsager's regression hypothesis, which in particular, permits to obtain the trap stiffness according to ${ }^{9}$ :

$$
\left(\kappa-\omega^{2} m\right)_{\omega_{S}}=\frac{2 k_{B} T}{P\left(\omega_{S}\right)} \frac{A_{P}}{\omega_{S} A_{S}} \sin (\Delta \phi)
$$

In the equation, $\mathrm{T}$ is the absolute temperature, $\mathrm{k}_{\mathrm{B}}$ Boltzmann's constant, $\mathrm{P}$ is the value of the unperturbed power spectrum at driving frequency $\omega$, and $A_{p}$ and $A_{s}$ are the amplitudes of the sinusoidal displacements of the particle and stage respectively. Finally, angle $\phi$ is the phase lag between these two oscillations and carries direct information about the relative importance of viscosity and elasticity in the medium. A value of $\phi$ close to $\pi / 2$ characterizes a predominantly viscous behavior whereas a value close to zero indicates the presence of strong elastic components. The method has been validated in F-actin gels and recently inside S. Pombe cells ${ }^{14}$.

We implemented the method and calibrated the volts-to-pN conversion factor (к. $\beta$ ) in two very different scenarios: in onion cells and in A549 cells (human lung epithelial adenocarcinoma). Our optical trapping and measurement setups have been described in detail elsewhere ${ }^{12,13}$. In short, a holographic optical trapping system was built around a Nikon Eclipse TE-2000 inverted microscope, which mounted a water-immersion objective (Nikon CFI, PlanApo 60x, NA = 1.2). The sample could be moved with a piezoelectric stage (Piezosystem Jena, TRITOR $102 \mathrm{SG}$ ) in a sinusoidal fashion with controlled amplitude and frequency (between 4-70 Hz). An oil-immersion, high-numerical aperture (NA=1.4) lens system was used to capture a large fraction of the scattered light. This light was finally directed to a duo-lateral position sensitive detector (PSD) for analysis.

Figure 3.a and 3.b illustrate the trapping arrangements. The A549 cell line is widely used as a model in the study of the pulmonary function and is composed of small $(\sim 15 \mu \mathrm{m})$, flat cells, which adhere easily to microscope slides without any further treatment. Cells were cultured on standard cover glasses, which were later used to build $\sim 100 \mu \mathrm{m}$-thick microchambers. These chambers containing cells and culture medium were mounted upside down on the piezo stage (Fig. 3.b) in such a way that the light scattered by the samples quickly reached the front lens of the measuring system, maximizing light collection. Optically dense vesicles showing directed motion (Figure 3.d), presumably lipid droplets, were easily seen under regular brightfield microscopy. The motion of these organelles could be abrogated by treating the cells with $5 \mu \mathrm{g} / \mathrm{mL}$ nocodazole, an agent which is known to disrupt microtubule polymerization, indicating that transport was mediated by molecular motors kinesin and dynein. The flat cells and high-optical contrast organelles produced patterns at the back-focal plane of the detection system (i.e. showed momentum spectra) which were similar to those produced by polystyrene microspheres suspended in water (Figure 3.e), indicating the absence of additional strong scattering structures along the light path in these cells. 


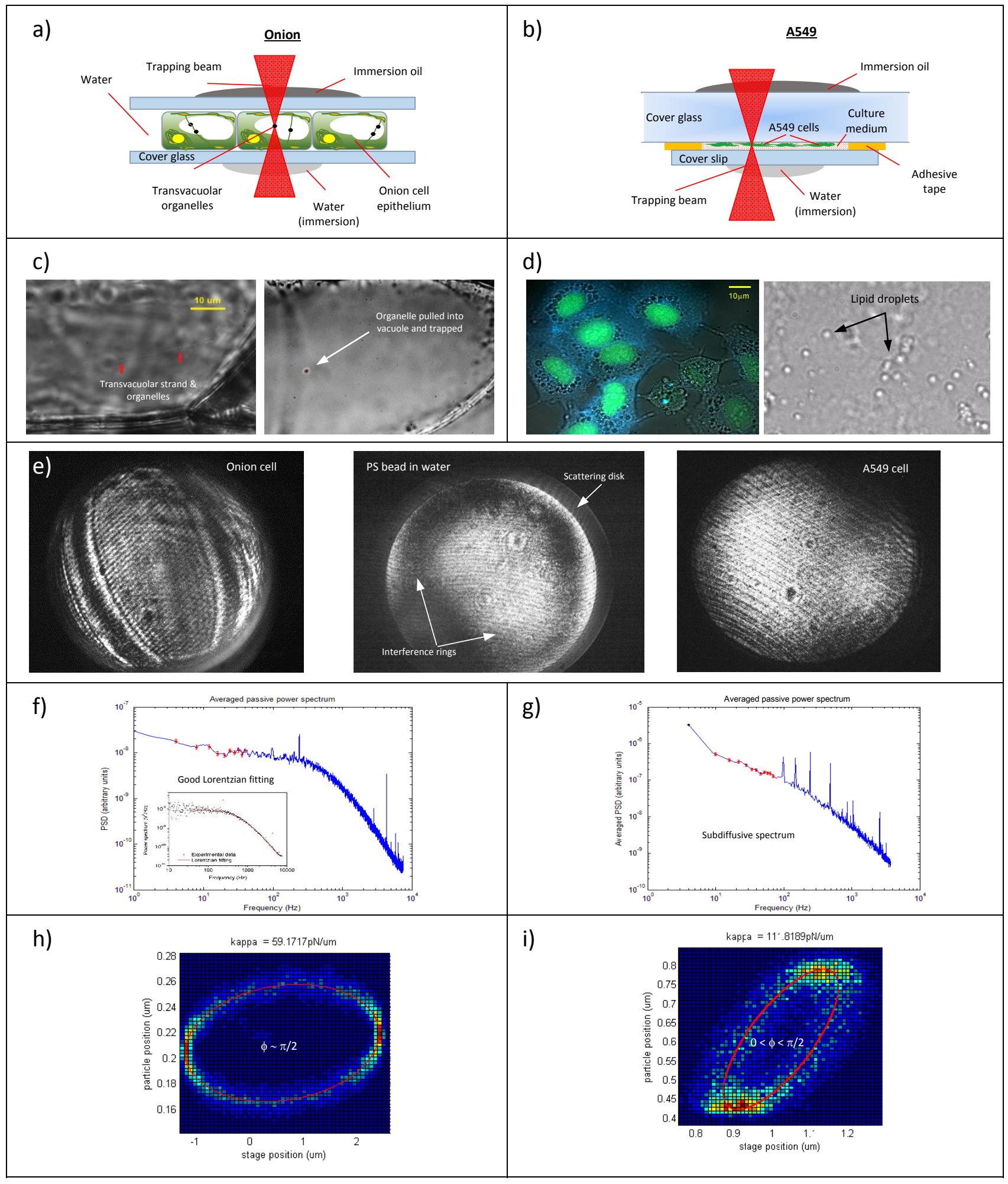

Figure 3. Calibration experiments were carried out in plant and mammalian cells. The obstacles to light propagation and the rheology of the trapping environment differed considerably between these two cell types. For a complete description of the figure, see text. 
On the contrary, the power spectrum of their Brownian fluctuations was clearly subdiffusive (Figure 3.g) and the phase difference between driving and induced oscillations smaller than $\pi / 2$ (Figure 3.i), both facts indicating a micromechanically complex cytoplasm with presence of important elastic elements.

On the other hand, plant cells are large $(\sim 50 \mu \mathrm{m})$ and are surrounded, in addition to by the lipid membrane, by a thick $(\sim 100 \mathrm{~nm}$ in onion cells), semi-rigid wall that gives them protection, rigidity and strength. A large part of our onion cells is occupied by a central vacuole, a membrane-bound organelle filled essentially with water. The vacuole is crossed by transvacuolar strands (Figure 3.c), tubular portions of the cytoplasm that connect the perinuclear region with the cortical cytoplasm. The transvacuolar strands contain cytoskeletal filaments that support organelle trafficking. We pulled with the optical trap some of these organelles out of the strand and into the vacuole, where they became freely suspended in the fluid, and then performed calibration experiments in this environment (Fig. 3.c). Contrary to the observations of the A549 cells, inside the vacuole of onion cells we observe a complex momentum structure at the back-focal plane of our instrument (Fig. 3.e), which indicates different obstacles in the path of light. However, the Brownian power spectrum is clearly Lorenztian (Fig. 3.f), typical of a viscous environment, as expected from the watery content of the vacuole. The phase difference between the stage and the induced motion on the organelle (i.e. during the active phase of the calibration) approaches $\pi / 2$ (Fig. 3.h), again indicating a purely viscous environment inside the vacuole.

These two experiments are thus complementary as they represent opposed scenarios:

1. Measurements based on active-passive calibrations should be easy in onion cells (almost no viscoelasticity or active processes) but demanding on the momentum method (large cells, obstacles in the packed cytoplasm and the presence of the cell wall).

2. The A549 cells should pose little problems to the momentum method (flat cells adjacent to the coverglass, little structure in the momentum distributions) but are demanding for the active-passive calibration (important viscoelasticity and possibility of ATP-dependent processes).

Our calibration results are summarized in Figure 4. In onion cells we performed sixty-six different experiments (Fig. 4.a). The plot shows that the calibration factor $\kappa . \beta$ is essentially constant (Fig. $4 . a$ ) although a $\sim 14 \%$ difference between the average $\kappa . \beta$ and $\alpha$ measured according to Equation 2 can be appreciated. Similarly, calibrations were carried out for 147 organelles in A549 (Fig. 4.b). Again, although the calibration product $\kappa . \beta$ is remarkably constant across experiments and does not depend on the trapped sample nor on local cytoplasmic conditions, there is a $\sim 16 \%$ difference with $\alpha$. The reason for this small discrepancy seems to be noise in the passive power spectrum at low frequencies. Each stiffness in Figure 4 was obtained as the average over ten different frequencies, in the range from 4-70 Hz. We have observed that the stiffnesses obtained at driving frequencies below $20 \mathrm{~Hz}$ are systematically lower by a $\sim 20 \%$ than stiffnesses obtained at higher frequencies (Fig. 5.a, the trap stiffness should not depend on the driving frequency).

a)

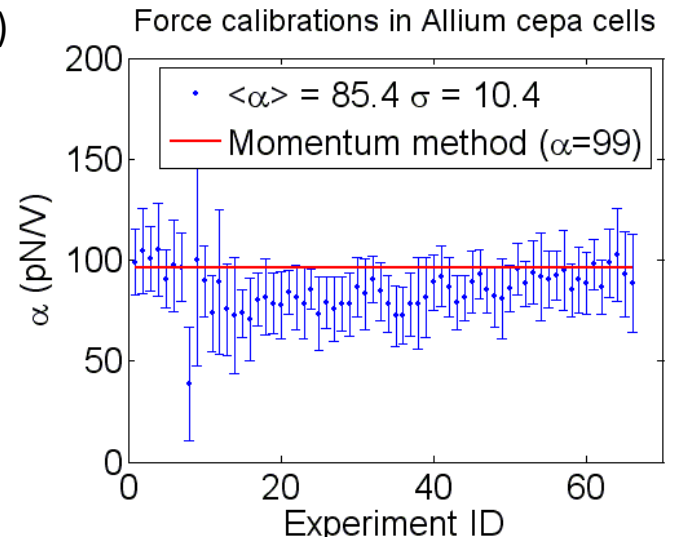

b)

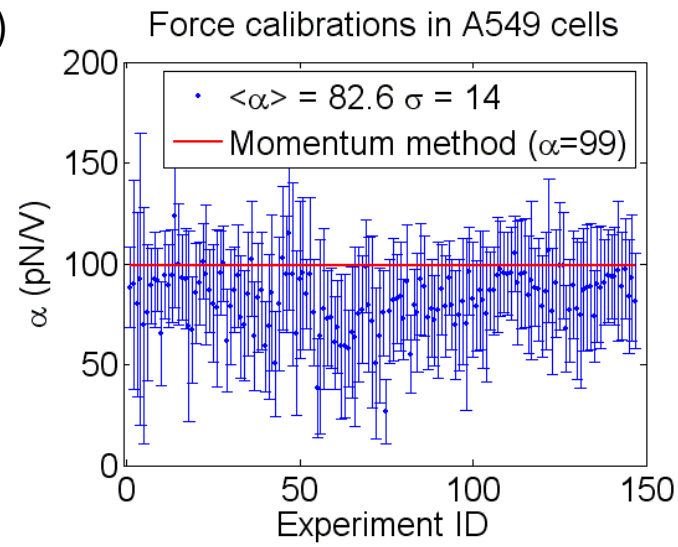

Figure 4. Comparison of calibration constant $\alpha$ measured according to equation $2(\alpha=99 \mathrm{pN} / \mathrm{V}))$ with the product $\kappa \beta$ measured through the active-passive method in a) onion (Allium cepa) and b) A549 cells. c) Fraction of the calibration results for the A549 cells with less than 25\% error (error bars are computed from instrumental errors and statistical errors following reference 14) 
a)

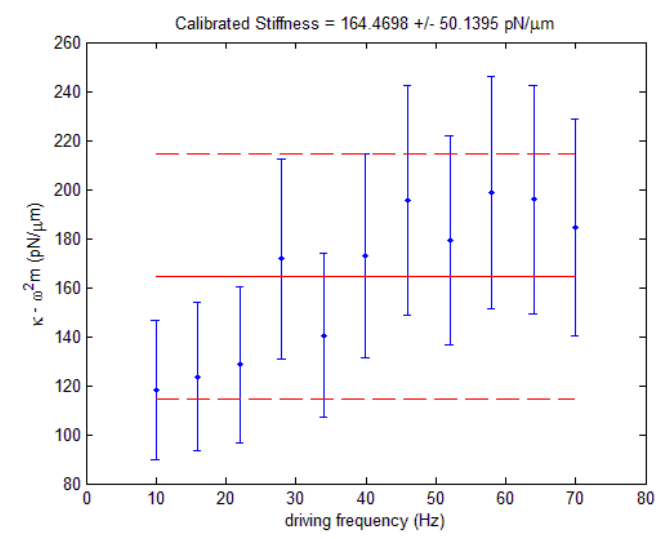

b)

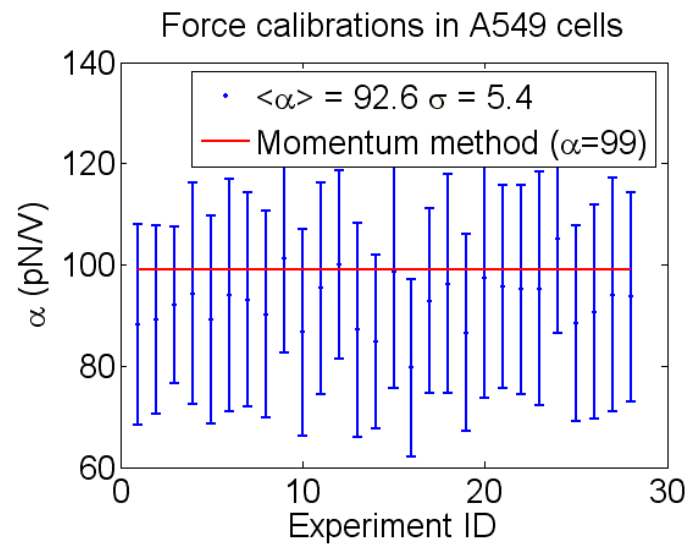

Figure 5. a) Typical behavior of the trap stiffness for different driving frequencies (the term $\omega^{2} \mathrm{~m}$ can be neglected ${ }^{8,9}$ ) in A549 cells. The graph systematically shows smaller values for lower frequencies. b) If we only plot the experiments with the smallest errors (less than 25\%) the discrepancy between these higher-quality calibrations and factor $\alpha$ becomes irrelevant.

A smaller stiffness can result from overestimated values of the passive spectrum at the corresponding driving frequency $\mathrm{P}\left(\omega_{\mathrm{s}}\right)$, according to Equation 5. Stage drifts or laser pointing fluctuations are especially present at small frequencies and can spuriously contribute to inflate the values of the thermal spectrum in this region, thus leading to an underestimation of the stiffness. This can be seen in the power spectrum of Figure 3.f, in which values below $22 \mathrm{~Hz}$ deviate from the Lorentzian plateau clearly. If we eliminate these problematic frequencies or alternatively, we choose from the experimental pool in Figure 4, those points having a smaller error (i.e. with stiffness having smaller standard deviations, see reference 14 for the error analysis) (Figure 5.b) the disagreement between the average k. $\beta$ and the calibration factor $\alpha$ deduced from first principles becomes statistically irrelevant, according to a t-student test with $99 \%$ confidence level.

As a consequence, these results indicate that our instrument is capable of keeping the calibration inside both types of cells, which indeed remains unaffected by scattering structures or a viscoelastic environment, and therefore show that the momentum method is suitable for performing force measurements in the cytoplasm of these cells without recalibration.

\section{MEASURING STALL FORCES OF KINESIN AND DYNEIN IN VIVO}

The momentum method therefore trades off experimental complexity: the instrument is more sophisticated and complex to build than a comparable system based on position measurements (i.e. back-focal plane interferometry) ${ }^{15}$ but once the system is up and running, the experiments become easier and more reliable, as no local calibrations are required according to our results above.

This allowed us to quickly make intracellular measurements of force. We focused on the A549 cell line as prior experiments using a variety of calibration approaches had shown discrepant stall force measurement of kinesin and dynein when compared to in vitro assays ${ }^{16,17}$. Following the procedures in these references, we identified lipid droplets moving in opposite directions, labeling them "inward" and "outward" depending on whether they were moving from the cell periphery towards the cell nucleus or vice versa, respectively. Inward-moving organelles are probably motored by dynein whereas outward-moving organelles are associated to kinesin motors. Representative force traces of stall events for these two directions are shown in Figure 6. The molecular motors associated to the trapped organelle exert increasing forces as they move away from the trap center. The velocity gradually decreases as the motor faces an increasing opposition from the trap, until the motor stalls (i.e the force trace shows a plateau). After a fraction of a second the motor protein dissociates from the microtubule and the optical trap brings the vesicle back to the center of 

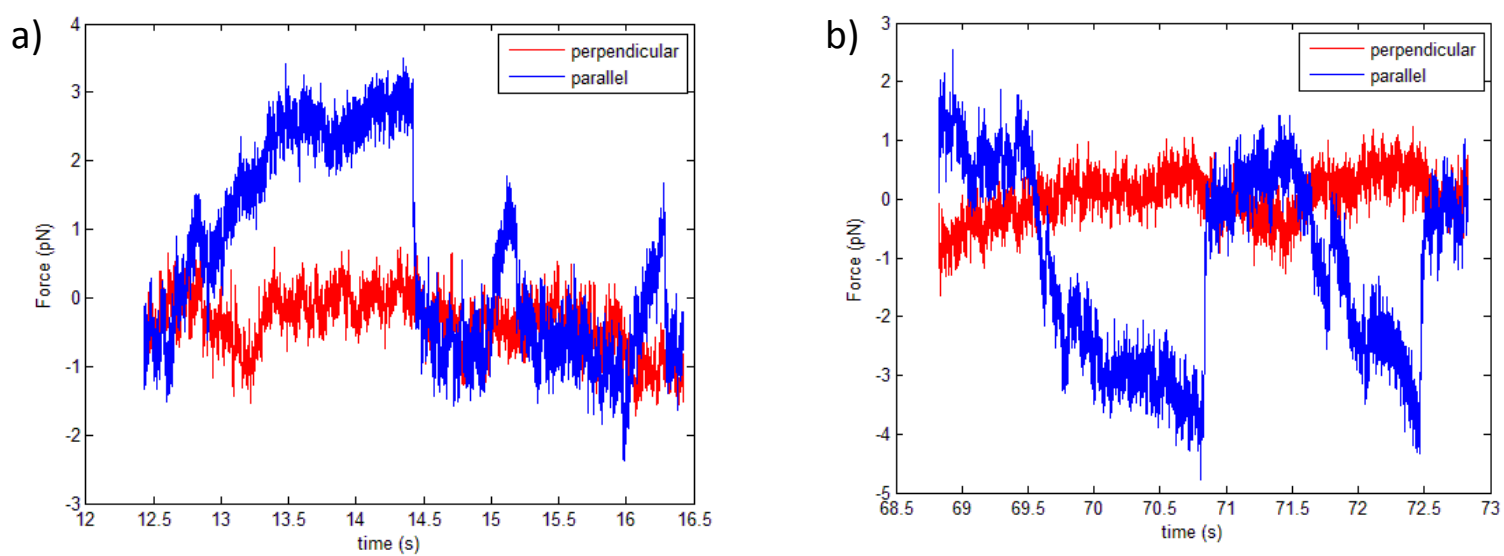

Figure 6. Typical force curves of stall events measured on a) inward-movig (dynein) vesicles and b) outward-moving (kinesin) vesicles.

the trap within milliseconds (the vertical jump in the force curve). The molecular motor can again rebind the microtubule and initiate a new run. Sometimes the molecular motor detaches from the cytoskeletal substrate before developing its maximum force (i.e. the smaller traces that can be seen in Fig. 6.a).

We registered many clear stall events where the motor reached a force plateau before dissociating in both the anterograde and retrograde directions. We discarded the experiments in which the perpendicular component of forces did not remain constant during the pull and release phases, which would indicate that the optical trap was not properly centered on the microtubule. A compilation (86 outward, 79 inward experiments) of stall forces for opposite-polarity motors is shown in Figure 7. Minus-directed motion (inward, dyein) shows a wide peak centered around $3.5 \mathrm{pN}$ and a secondary peak between $7-10 \mathrm{pN}$. The graph corresponding to the plus-directed motion (kinesin) shows clear peaks at $\sim 3.5$ and $\sim 6.5 \mathrm{pN}$. These results are in agreement with those recently reported ${ }^{16,18}$ that show comparable unitary forces for kinesin and dynein in vivo. These values differ significantly from those found in vitro ( $4-7 \mathrm{pN}$ for kinesin and $\sim 1.1 \mathrm{pN}$ for dynein).

a)

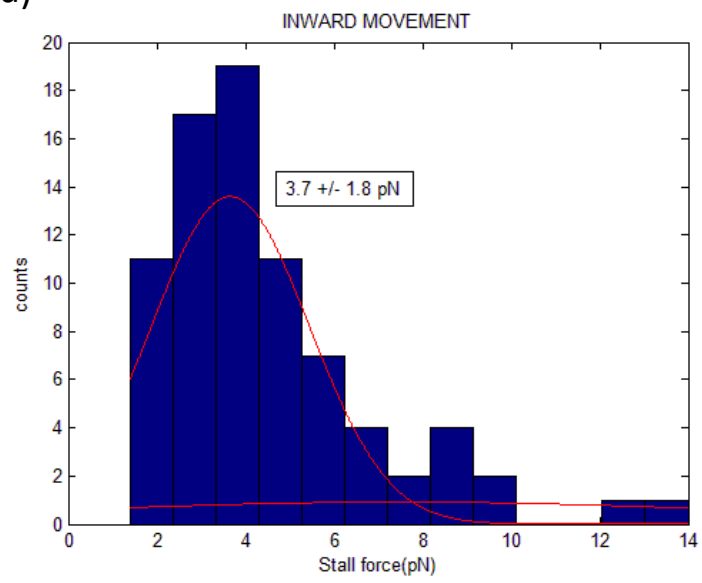

b)

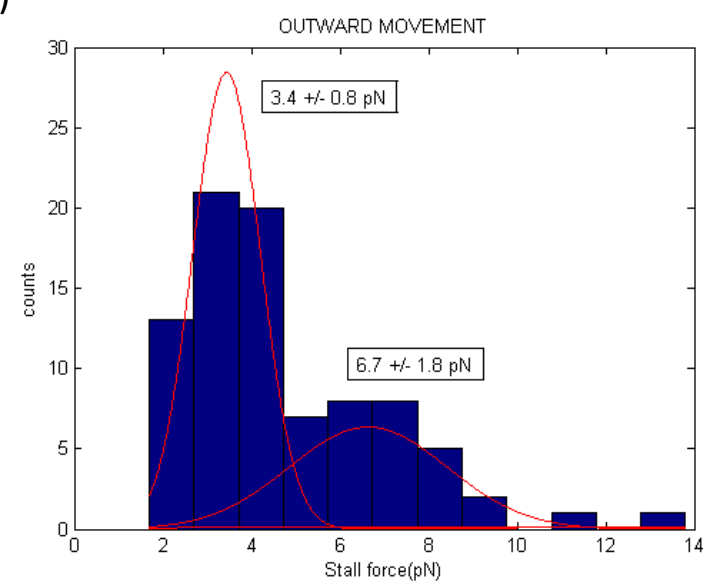

Figure 7. Histogram of stall force measured on a) inward-movig (dynein) vesicles and b) outward-moving (kinesin) vesicles. 


\section{REFERENCES}

[1] Svoboda, K., Schmidt, C. F., Schnapp, B. J., and Block, S. M., "Direct observation of kinesin stepping by optical trapping interferometry," Nature, 365(6448), 721-727 (1993).

[2] Dogterom, M., and Yurke, B., "Measurement of the force-velocity relation for growing microtubules," Science, 278(5339), 856-860 (1997).

[3] Bar-Ziv, R., Moses, E., and Nelson P., "Dynamic excitations in membranes induced by optical tweezers," Biophys. J. 75, 294-320 (1998).

[4] Rai, A. K., Rai, A., Ramaiya, A. J., Jha, R., and Mallik, R., "Molecular adaptations allow dynein to generate large collective forces inside cells," Cell, 152(1), 172-182 (2013).

[5] Mallik, R., Carter, B. C., Lex, S. A., King, S. J., and Gross, S. P.,"Cytoplasmic dynein functions as a gear in response to load," Nature, 427(6975), 649-652 (2004).

[6] Toba, S., Watanabe, T. M., Yamaguchi-Okimoto, L., Toyoshima, Y. Y., and Higuchi, H., "Overlapping handover-hand mechanism of single molecular motility of cytoplasmic dynein," Proceedings of the National Academy of Sciences, 103(15), 5741-5745 (2006).

[7] Holzbaur, E. L., and Goldman, Y. E., "Coordination of molecular motors: from in vitro assays to intracellular dynamics," Current opinion in cell biology, 22(1), 4-13 (2010).

[8] Fischer, M., and Berg-Sørensen, K., "Calibration of trapping force and response function of optical tweezers in viscoelastic media," Journal of Optics A: Pure and Applied Optics, 9(8), S239 (2007).

[9] Fischer, M., Richardson, A. C., Reihani, S. N. S., Oddershede, L. B., and Berg-Sørensen, K., "Active-passive calibration of optical tweezers in viscoelastic media," Review of Scientific Instruments, 81(1), 015103(2010).

[10] Smith, S. B., Cui, Y., and Bustamante, C., "Overstretching B-DNA: the elastic response of individual doublestranded and single-stranded DNA molecules," Science, 271(5250), 795-799 (1996).

[11] Smith, S. B., Cui, Y., and Bustamante, C., "Optical-trap force transducer that operates by direct measurement of light momentum,” Methods Enzymol. 361, 134-162 (2003).

[12] Farré, A., and Montes-Usategui, M., "A force detection technique for single-beam optical traps based on direct measurement of light momentum changes," Optics Express, 18(11), 11955-11968 (2010).

[13]Farré, A., Marsà, F., and Montes-Usategui, M., "Optimized back-focal-plane interferometry directly measures forces of optically trapped particles," Opt. Express 20, 12270-12291 (2012).

[14] Mas, J., Richardson, A. C., Reihani, S. N. S., Oddershede, L. B., and Berg-Sørensen, K., "Quantitative determination of optical trapping strength and viscoelastic moduli inside living cells," Physical biology, 10(4), 046006 (2013).

[15]Farré, A., Marsà, F., Montes-Usategui, M., “A force measurement instrument for optical tweezers based on the detection of light momentum changes," this same volume.

[16] Sims, P. A., and Xie, X. S., "Probing dynein and kinesin stepping with mechanical manipulation in a living cell," ChemPhysChem, 10(9-10), 1511-1516 (2009).

[17]Blehm, B. H., Schroer, T. A., Trybus, K. M., Chemla, Y. R., and Selvin, P. R., "In vivo optical trapping indicates kinesin's stall force is reduced by dynein during intracellular transport," Proceedings of the National Academy of Sciences, 110(9), 3381-3386 (2013).

[18] Leidel, C., Longoria, R. A., Marquez Gutierrez, F., and Shubeita, G. T., "Measuring molecular motor forces in vivo: implications for tug-of-war models of bidirectional transport," Biophys. J. 103, 492-500 (2012). 